\title{
Treatment patterns, health care resource utilization and costs of rheumatoid arthritis patients in Italy: findings from a retrospective administrative database analysis
}

This article was published in the following Dove Press journal:

Open Access Rheumatology: Research and Reviews

\author{
Walid Fakhouri' \\ Pedro Lopez-Romero² \\ Silvia Antonelli ${ }^{3}$ \\ Serena Losi $^{4}$ \\ Veronica Rogai ${ }^{4}$ \\ Stefano Buda ${ }^{5}$ \\ Diego Sangiorgi ${ }^{5}$ \\ Valentina Perrone ${ }^{5}$ \\ Luca Degli Esposti \\ 'Eli Lilly \& Company, Erl Wood, \\ UK; ${ }^{2}$ Eli Lilly \& Company, Madrid, \\ Spain; ${ }^{3}$ Eli Lilly Italy S.p.A., Roma, \\ Italy; ${ }^{4}$ Eli Lilly Italy S.p.A., Sesto \\ Fiorentino, Italy; ${ }^{5} \mathrm{Cli}$ Con S.r.l. Health \\ Economics \& Outcomes Research, \\ Ravenna, Italy
}

Objectives: This study aimed to: 1) describe treatment patterns and drug utilization profile (in terms of therapeutic strategy used, switch, persistence and drug consumption variation) among adult patients affected by rheumatoid arthritis (RA), and 2) estimate the health care resource utilization and its associated direct cost for the management of RA patients.

Methods: A retrospective cohort analysis, using administrative databases of six Local Health Units in Italy, was performed. All adult patients with a confirmed diagnosis of RA between January 1, 2010 and December 31, 2014 were enrolled. The date of the first RA diagnosis according to the study criteria during the study period represented the index date (ID) for each patient. Patients enrolled were observed from the ID for at least 12 months (follow-up period), and their clinical characteristics were investigated for 12 months prior to the ID.

Results: A total of 10,401 patients with a confirmed RA diagnosis were included. Mean age was 63.0 years and $25 \%$ were male; $67 \%$ of patients were untreated at ID. During the followup period, $67.8 \%$ of patients treated with biologic agents were persistent with initial therapy, compared to $45.7 \%$ for patients on conventional synthetic disease-modifying antirheumatic drugs (csDMARDs), while $11 \%$ of patients treated with biologic agents switched during the follow-up period, compared to $17.6 \%$ of csDMARDs-treated ones. At the end of the follow-up period, $14.7 \%$ of all patients in the analysis had an increase and $12.6 \%$ of them had a decrease in their initial drug consumption. The mean cost per RA patient was $€ 3,743$.

Conclusion: Our study showed that there is still much that needs to be learned about the prescription of csDMARDs and biologics to RA patients in Italy and to identify areas for future research. The knowledge of RA management in a real-life clinical setting could offer an opportunity to improve the management of RA in Italy.

Keywords: rheumatoid arthritis, treatment patterns, drug utilization, real-world data

\section{Introduction}

Rheumatoid arthritis (RA) is an autoimmune disease that causes chronic joint inflammation. ${ }^{1}$ RA is one of the most common rheumatic diseases in the world. ${ }^{2}$ The global prevalence of RA varies between $0.3 \%$ and $1 \% .{ }^{1}$ Worldwide, the annual incidence of RA is $~ 3$ cases per 10,000 population and it tends to start between the ages of 35 and 50 years. Women are affected by RA about three times more often than men are, but sex differences diminish in older age groups. ${ }^{1,3}$ One recent epidemiological study conducted in almost five million Italian people aged $\geq 18$ years, representative of the entire Italian population, reported an RA prevalence of $41 \%$ and incidence of 35 new cases per 100,000 adult patients per year. ${ }^{3}$
Correspondence: Luca Degli Esposti CliCon Srl, Health, Economics and Outcomes Research, Via Salara, 36, 48100 Ravenna, Italy

Tel +3954438393

Fax +39544212699

Email luca.degliesposti@clicon.it 
The management of RA according to the European League Against Rheumatism (EULAR ${ }^{4,5}$ recommendations includes a number of different treatments options, but there is no cure. Optimal care for patients with RA consists of an integrated approach that includes both pharmacologic and non-pharmacologic therapies. Medication-based therapies comprise several classes of agents. Some are used primarily to ease the symptoms of RA, while others are used to slow or stop the course of the disease and to inhibit structural damage.

Nonsteroidal anti-inflammatory drugs (NSAIDs) are used for pain relief as well as reducing inflammation (i.e., ibuprofen and naproxen sodium). Corticosteroid medications, such as prednisone and methylprednisolone, are effective at reducing inflammation and pain and slowing joint damage. They are usually recommended when NSAIDs have not helped. RA patients are also prescribed disease-modifying antirheumatic drugs (DMARDs). These drugs may slow down the progression of RA and save the joints and other tissue from permanent damage. DMARDs form two major classes: conventional synthetic compounds (csDMARDs) and biological agents. Although biologic drugs are technically a type of DMARD, they are usually just called biologics, which distinguishes them from csDMARDs.

The most commonly used csDMARDs in the treatment of RA patients are methotrexate, leflunomide, hydroxychloroquine and sulfasalazine. In terms of biologics, patients can be prescribed any of the following treatments: tumour necrosis factor inhibitors (adalimumab, certolizumab pegol, etanercept, golimumab and infliximab), T-cell costimulation inhibitor (abatacept), anti-B-cell agent (rituximab), interleukin (IL)-6 receptor blocking monoclonal antibody (tocilizumab), as well as IL-1 inhibitor (anakinra). These drugs can target different signaling pathways of the immune system that trigger inflammation that causes joint and tissue damage. A new subcategory of DMARDs known as "JAK inhibitors" blocks the Janus kinase (JAK) signaling pathways, which are involved in the body's immune response.

The treatment armamentarium for RA has substantially expanded in the last decades, and there is a wealth of information on the efficacy and safety of various RA treatments coming from randomized clinical trials. However, there is still need for more evidence from routine clinical practice on which patient is being prescribed which drug, and what impact this has on the health care system in terms of health care resource use and costs.

RA affects patients during the early or middle years of life, and given the progressive nature of the disease, many patients will experience worsening of their symptoms over time. This has major implications for the patient's long-term overall health and function. Consequently, the high level of disability associated with RA leads to a considerable social and economic cost.

The recent Global Burden of Disease 2010 study report showed that, of the 291 conditions studied, RA was ranked 74th in terms of burden as measured by disability-adjusted life years, and $42 \mathrm{nd}$ in terms of disability as measured by years of life lived with disability. ${ }^{3}$ In Italy specifically, a recent modeling analysis based on Monte Carlo simulation of the annual economic burden of RA estimated that, in 2015, the economic burden associated with RA was $\sim € 2.0$ billion, with a confidence interval (CI) of $95 \%$ ranging from $€ 1.78$ to $€ 2.3$ billion. ${ }^{7}$ Almost half of the expenditure (45\%) was due to indirect costs ( $95 \% \mathrm{CI} € 0.8-1.0$ billion); $45 \%$ depended on direct medical costs ( $95 \% \mathrm{CI} € 0.7-1.1$ billion), and the residual $10 \%$ was determined by direct non-medical costs (95\% CI $€ 0.16-0.25$ billion). ${ }^{7}$

To gain a comprehensive understanding, from a realworld perspective, about the current use of RA treatments in Italy, we conducted a study with the following two main objectives: 1) to describe treatment patterns and drug utilization profile (in terms of therapeutic strategy used, switch, persistence and drug consumption variation) among adult patients affected by RA, and 2) to estimate the health care resource utilization and its associated direct cost for the management of RA patients.

\section{Methods}

\section{Data sources}

This study was conducted using administrative databases (DBs) of six Italian Local Health Units (LHUs) geographically distributed throughout the national territory (Verona, Veneto; Pavia, Lombardy; Roma A, Lazio; Lecce, Apulia; Grossetto, Tuscany; and Caserta, Campania), for a total number of about 3,480,000 health-assisted individuals.

The following databases are used in this study: "Beneficiary' DB", which contains patient demographic data; "Pharmaceuticals DB", which includes information for each medication prescription, such as the prescribing physician's number and the Anatomical-Therapeutic-Chemical (ATC) code of the drug; "Payment exemption DB", which contains the records of all disease exemptions, including the exemption code (identifying the disease for which the exemption was granted); "Hospitalization DB", which includes information on discharge for each hospitalization, in particular the date of admission and discharge, main and secondary diagnosis, coded according to the International 
Classification of Diseases, Ninth Revision, Clinical Modification (ICD-9-CM) and Diagnosis-Related Group (DRG) reimbursement rate; and finally "Diagnostic tests and specialist visits DB", which includes all information about outpatient specialist services.

The data were extracted by each LHU, and their databases were anonymized in full compliance with the Italian code of protection of personal data (Legislative Decree, 196/03). To guarantee patient privacy, each subject was assigned an anonymous unique patient code. The patient code in each database permitted electronic linking across all databases. No identifiers related to patients were provided to the researchers. All the results of the analyses were produced as aggregated summaries, which are not possible to assign, either directly or indirectly, to the individual patients. Informed consent was not required for using encrypted retrospective information. This study was notified to the local ethics committee in each participating LHU according to the Italian law regarding the conduct of observational analysis, and the LHU Ethics Committees approved the study. ${ }^{8}$

\section{Cohort definition}

This was an observational retrospective cohort study, which included all patients aged $\geq 18$ years with a confirmed or suspected diagnosis of RA between January 1, 2010 and December 31, 2014 ("identification period"). Confirmed RA patients were identified as such by treatment with RA-specific drugs (e.g., biologic agents, csDMARDs and corticosteroids), and hospitalization with a primary or secondary diagnosis of RA (ICD-9-CM code: 714.0) or specific exemption code (code: 006). Suspected RA cases were identified by at least one prescription for RA medications.

The date of the first RA diagnosis according to the study criteria during the "identification period" represented the "index date" for each patient. All patients were stratified by the type of the diagnosis "Confirmed RA" or "Suspected RA" at "index date". The analyses were conducted only on the cohort of patients with "Confirmed RA". The decision to exclude the "Suspected RA" cases from the analyses was based on the potential bias the inclusion of these patients may have on the results (patients could have been prescribed one of the specific RA drugs but for another chronic inflammatory rheumatic disease).

Patients enrolled were observed from the index date for at least 12 months (follow-up period), and their clinical characteristics were investigated for 12 months prior to the "index date" (characterization period).
Patients who have moved to other LHUs during the follow-up period and/or with concomitant pathologies (such as ankylosing spondylitis, psoriatic arthritis, psoriasis, Crohn's disease) were excluded from the analysis. We identified ankylosing spondylitis and Crohn's disease from hospital admissions or exemption codes.

Baseline information, including demographics characteristics, hospital admissions, prescribed drugs, and comorbidities, was collected. The treatments of interest were: biological treatments (abatacept [ATC code: L04AA24], etanercept [ATC code: L04AB01/L04AA11], infliximab [ATC codes: L04AB02, L04AA12], rituximab [ATC code: L01XC02], adalimumab [ATC codes: L04AB04, L04AA17], certolizumab [ATC code: L04AB05], golimumab [ATC codes: L04AB06, H05BX01], tocilizumab [ATC code: L04AC07]) and csDMARDs (methotrexate [ATC codes: L01BA01, L04AX03], ciclosporin [ATC codes: L04AD01, S01XA18], leflunomide [ATC code: L04AA13], sulfasalazine [ATC code: A07EC01], acitretin [ATC code: D05BB02]). The use of csDMARDs and biologic agents during the characterization period was also evaluated. Naïve RA patients were defined as those who had not received any RA-related treatment (considering both csDMARDs and biologic agents) before the year preceding the index date. Among them, we defined RA patients as biologic-naïve patients if they had not received any biologic agent during the year preceding the index date.

Comorbidity profiles were measured using the Charlson Comorbidity Index (CCI),${ }^{9}$ which assigns a score to each concomitant disease identified through treatments and hospitalizations during the characterization period. The CCI score reflects the patient's overall health status.

According to the therapeutic strategy used at index date (csDMARDs or biologics), the pharmaco-utilization profiles of patients (in terms of persistence, switch rate and drug consumption) were estimated during the follow-up period.

Patients were classified as persistent if they were still on treatment with the index drug during the last 3 months of observation. A switch was defined as the presence of a different therapy other than that administered at the index date during the follow-up period. Drug consumption, escalation or reduction was evaluated as the change in the average dose prescribed between the two following prescriptive intervals during the period of observation. Dose escalation or reduction was defined as having two consecutive prescriptions with an average weekly dose $30 \%$ greater or lower than the initial average weekly dose, in line with previously published 
methods. ${ }^{10,11}$ Variations in drug consumption were calculated only for patients naïve to biologics and persisting with initial treatment during the follow-up period.

In order to estimate RA management costs, the overall utilization of health care resources was assessed in the 12 months after index date (follow-up period). Health care utilization in terms of hospitalizations, drug treatments, and diagnostic tests were classified, in the analysis, as related and not related to RA. These were evaluated and expressed as an average value per patient, considering RA treatments (biologic agents, csDMARDs and/or corticosteroids [ATC code: H02]), hospitalizations inherent to RA (ICD-9-CM code: 714 ) and all outpatient specialist services (both related and not related to RA) during the follow-up period.

\section{Cost analysis}

Health care resource utilization and costs were assessed as an average per patient per year. Costs for each single direct health resource used in the follow-up period was evaluated. Drug costs were evaluated using the Italian National Health Service (NHS) purchase price. Hospitalization costs were determined using the DRG tariff. The cost of instrumental and laboratory examinations was defined according to the tariffs applied by the regions. The cost analysis was conducted from the perspective of the Italian NHS. Costs were reported in Euros (€) currency.

\section{Statistical analysis}

The analyses were descriptive, and no formal statistical comparisons were performed. Continuous variables were reported as mean and standard deviation (SD), whereas categorical variables were expressed as numbers and percentages. All statistical analyses were performed using STATA SE version 12.0 (StataCorp LP, College Station, TX, USA), version 12.0. Data management was carried out using Microsoft SQL Server 2012.

\section{Results}

Of the total population of 3,480,000 health-assisted subjects, 32,344 were patients with suspected or confirmed RA diagnosis, based on the inclusion criteria of the study. Of those, 10,401 patients had a confirmed diagnosis of RA and constituted the sample of patients on whom the analyses were conducted. Figure 1 shows details of the flowchart of cohort definition.

In terms of demographic characteristics, the mean (SD) age at index date was 63.0 (14.8) years. A majority of the patients were female. In terms of clinical characteristics, almost three-quarters $(72 \%, n=7,452)$ of patients had one or more comorbidity at baseline (index date); the mean (SD) CCI score was 1.5 (1.6). Sixty-seven percent $(n=6,959)$ of patients had not received any RA-related treatment (considering both csDMARDs and biologic agents) before the index

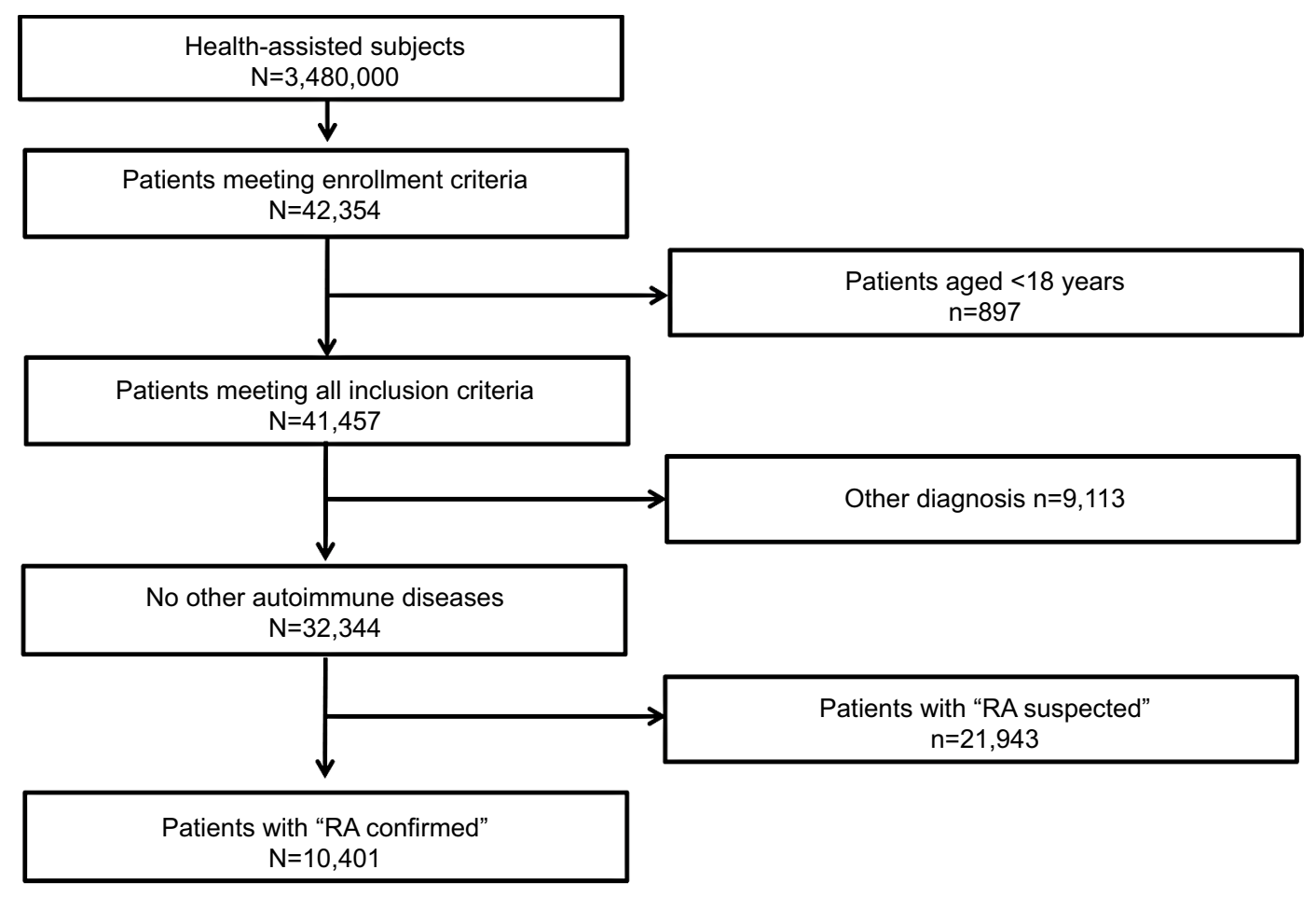

Figure I Flowchart of cohort definition. Abbreviation: RA, rheumatoid arthritis. 
date, while $83 \%(n=8,619)$ and $3 \%(n=301)$ were prescribed a csDMARD and biologic agents at baseline, respectively. Of patients treated with biologic agents, 105 out of $301(1 \%$ of all patients included in the analysis) were biologic-naïve at baseline; among these, $30.5 \%(n=32)$ of patients received csDMARDs during the characterization period. Baseline demographic, therapeutic and clinical characteristics of patients included in the study are shown in Table 1.

The results on health care resource utilization are reported in Figure 2. Drug utilization profile during the follow-up period, stratified according to the therapeutic strategy that patients were receiving at baseline, is reported in Figure 3.

Table I Demographic, therapeutic and clinical characteristics of patients included in the study at index date

\begin{tabular}{ll}
\hline & Total $\mathbf{n}(\%)$ \\
\hline $\mathrm{N}$ & $10,40 \mathrm{I}$ \\
Age, years (mean $\pm \mathrm{SD}$ ) & $63.0 \pm 14.8$ \\
Male & $2,608(25)$ \\
Charlson Comorbidity Index (mean $\pm \mathrm{SD})$ & $1.5 \pm 1.6$ \\
Charlson Comorbidity Index $\geq \mathrm{I}$ & $7,452(72)$ \\
Previously untreated & $6,959(67)$ \\
Patients who took at least one csDMARD at & $8,619(82.9)$ \\
baseline & \\
Patients who took two or more csDMARDs at & $652(6.3)$ \\
baseline & \\
Biologics & $301(2.9)$ \\
Biologic-naïe & $105(1.0)$ \\
\hline
\end{tabular}

Note: Patients who were not treated with biologic agents before the index date were defined untreated.

Abbreviations: csDMARDs, conventional synthetic disease-modifying antirheumatic drugs; SD, standard deviation.
The analyses showed that $67.8 \%(n=204)$ of patients treated with biologic agents were persistent with initial therapy, compared to $45.7 \%(n=3,939)$ for patients on csDMARDs. At 12 months, patients treated with biologic agents were found to have a lower switch rate from initial therapy compared to patients on csDMARDs $(11.0 \%$ [n=33] and $17.6 \%$ $[n=1,517]$, respectively). The analysis of drug consumption variation showed that, at the end of the follow-up period, $14.7 \%(n=1,529)$ of all patients in the analysis had an increase and $12.6 \%(n=1,311)$ of them had a decrease in their initial drug consumption.

The average annual health care costs for the management of RA patients, based on health care resource utilization from index date and through the follow-up period, are reported in Figure 4 . The mean cost per RA patient was $€ 3,743$, of which $€ 687$ was cost of RA-related drugs and $€ 882$ was cost of RA-related hospitalizations, while mean cost per patient for drugs and hospitalizations that were not related to RA were $€ 778$ and $€ 857$, respectively. The mean cost per patient for use of outpatient specialist services was $€ 540$.

\section{Discussion}

In the present study we evaluated, in an Italian real-world setting, treatment patterns and drug utilization profile among adult patients diagnosed with RA, and we estimated the health care resource use and direct costs for the management of RA patients using data from routine clinical practice.

The analyses were done retrospectively in an unselected Italian patient population in routine clinical practice. They

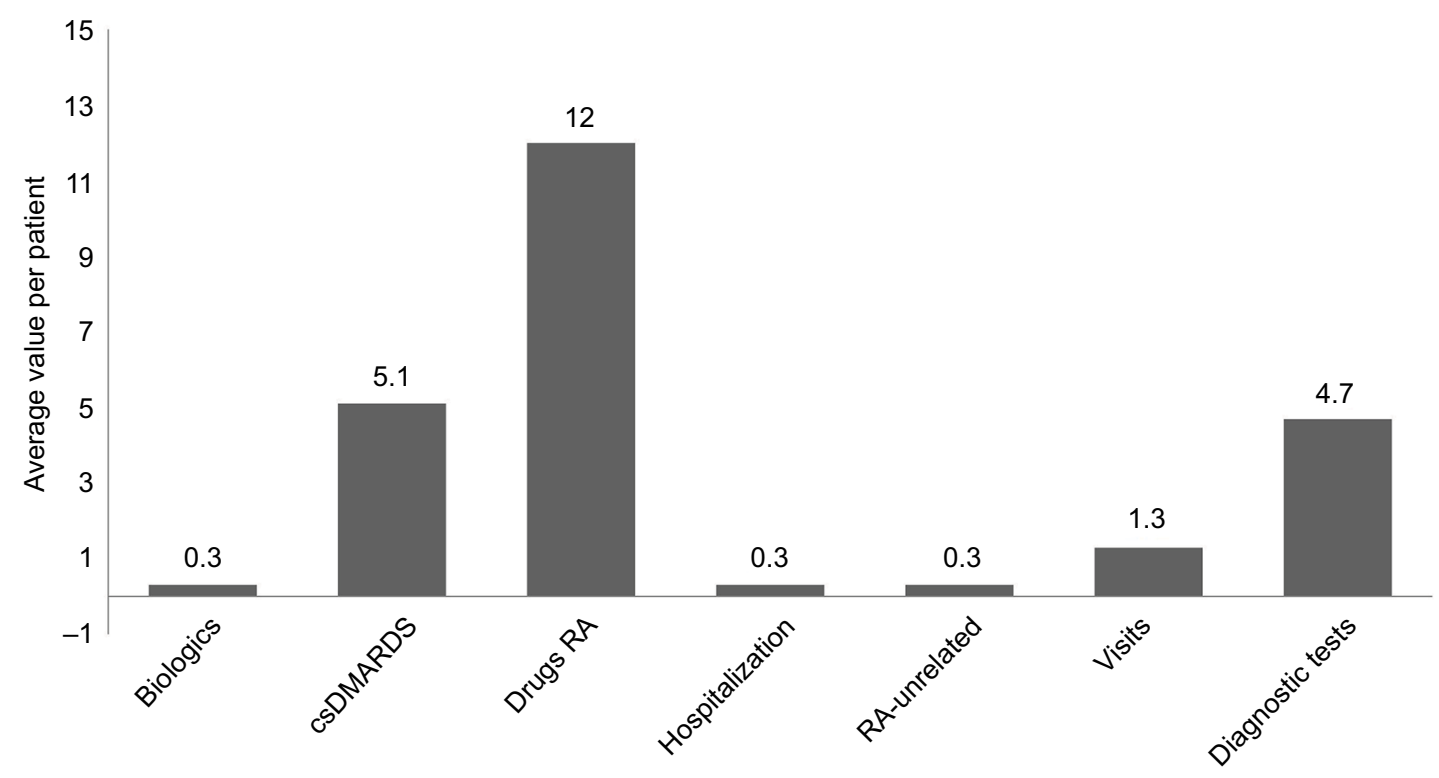

Figure 2 Health care resource consumption during the follow-up period ( 12 months).

Abbreviations: csDMARDs, conventional synthetic disease-modifying antirheumatic drugs; RA, rheumatoid arthritis. 


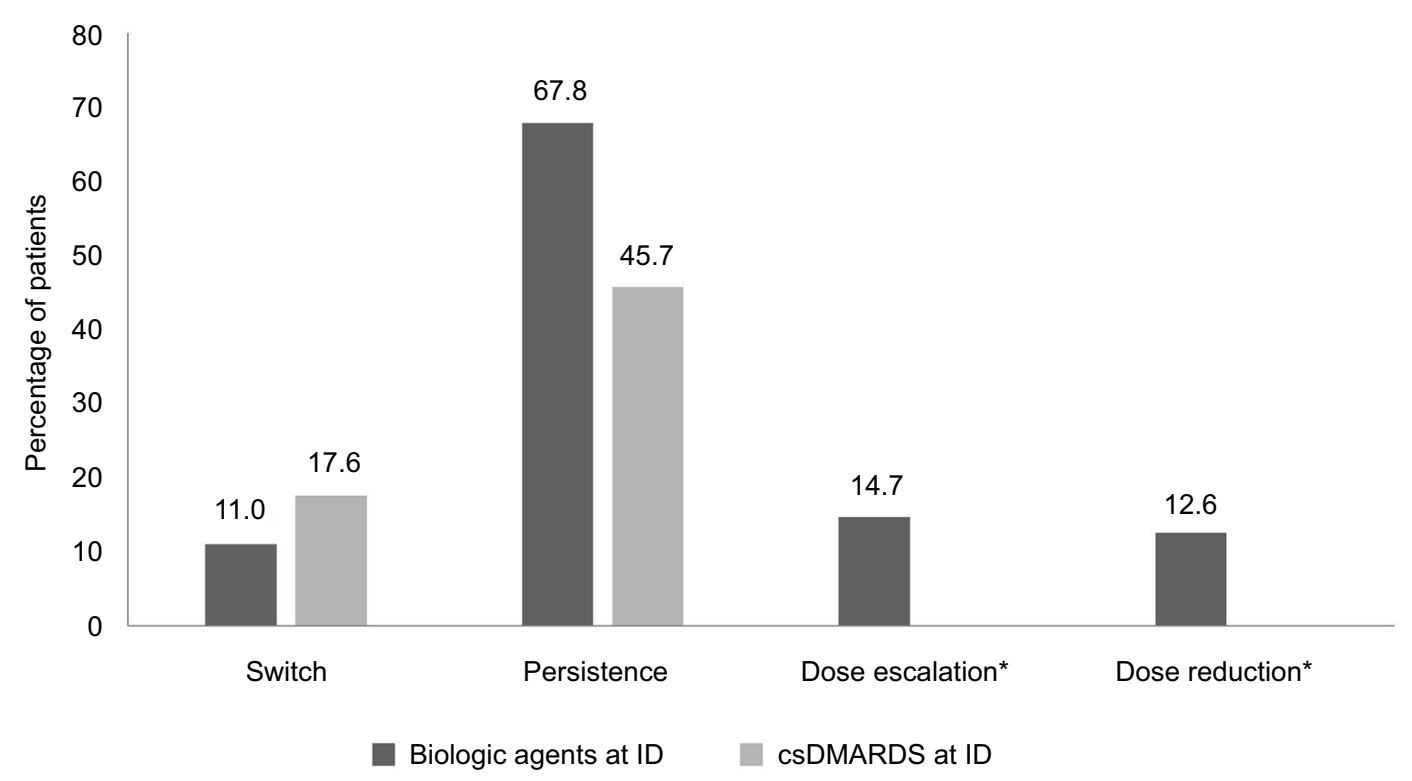

Figure 3 Drug utilization profile during the follow-up period, stratified according to the therapeutic strategy at baseline (index date). Note: *Patients naïve to biologic agents at index date $(\mathrm{N}=32)$.

Abbreviations: csDMARDs, conventional synthetic disease-modifying antirheumatic drugs; ID, index date.

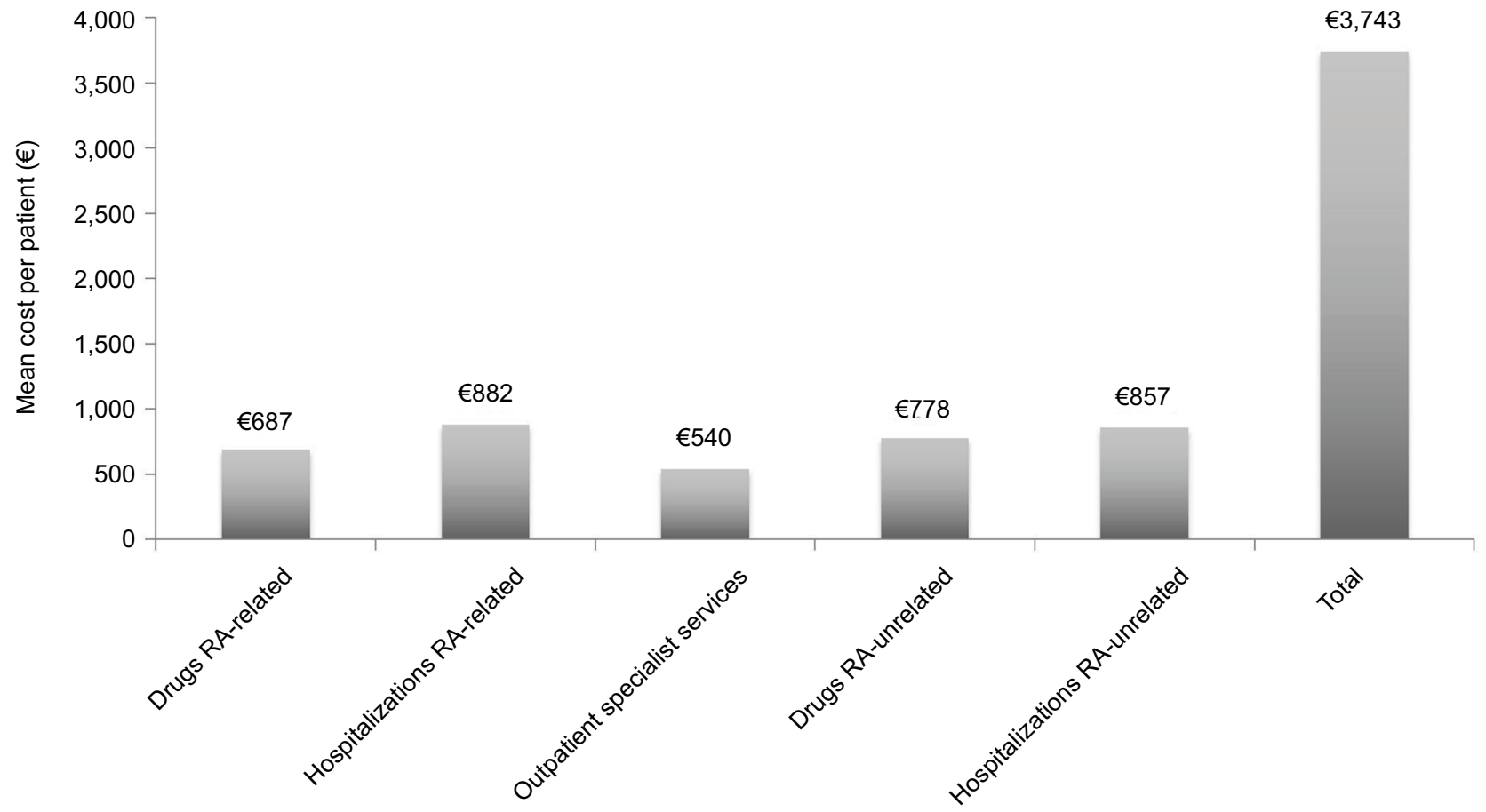

Figure 4 The annual health care resource costs per patient during the follow-up period ( 12 months). Abbreviation: RA, rheumatoid arthritis.

showed that about $0.3 \%$ of all health-assisted subjects in the LHUs who took part in the study had a confirmed diagnosis of RA and had been undergoing chronic treatment with specific RA drugs. Patients with other chronic inflammatory rheumatic diseases, who might also have been taking one of the specific RA drugs, were excluded from our analysis to prevent bias in the results. Our results about prevalence are similar to those reported in other Italian studies ${ }^{3,11-14}$ with values ranging from $0.38 \%$ to $0.48 \%$.

Despite that RA treatment has made major advances over the past few decades, especially with the introduction of biologics as a treatment option for RA patients, the majority of the 
patients in our study were found to be on csDMARDs rather than biologics. This may be due to the patients in the study having had less severe RA or a state of low disease activity that warranted no treatment with biologic agents. It could also be that patients may still have been kept on csDMARDs despite not achieving remission or low disease activity as recommended in the RA guidelines. ${ }^{4}$ Given that administrative claims databases do not collect clinical data on effectiveness or disease activity, we were not able to evaluate these hypotheses.

Our analyses indicated a higher level of persistence in RA patients treated with biologic therapies in comparison to those treated with csDMARDs (67.8\% vs $45.7 \%)$. The results presented here are consistent with other analyses conducted in the real-world setting. ${ }^{15-17}$ It is important to note that the variation estimates in previous research may be due to the method of calculating persistence to therapy. Lower persistence and adherence may indicate deficient drug utilization and are often correlated with a greater extent of unrealized treatment benefit and poorer clinical outcomes in RA. Previous studies in the real-world clinical setting have suggested that some patients need an upward dose adjustment of medication to achieve or maintain a sufficient clinical response, ${ }^{15,16,18}$ both in patients who are not likely to benefit from intensified therapy and in patients who have had an inadequate response to treatment or intolerable adverse events. The guidelines in Italy recommend switching to another treatment with another mode of action in those instances. ${ }^{19}$

Our study showed that patients on biologics (11\%) were less likely to switch treatment during the follow-up period in comparison to patients on csDMARDs (17.6\%), while the percentage of patients naïve to biologics and persisting with initial treatment who had dose escalation or dose reduction during the follow-up period was $14.7 \%$ and $12.6 \%$, respectively. Our findings support the limited evidence from real-world studies that assessed dose variations. ${ }^{16}$ The analyses on persistence and switching highlight the need for a deeper understanding of the reasons why most patients were on csDMARDs despite the lower persistence rates with these treatments, and to identify in a real-world setting whether or not lower persistence levels were associated with reduced clinical benefit in that population, and whether or not these patients would have benefited from being given a biologic treatment earlier.

We also assessed the cost of management of RA patients treated with biologic agents or csDMARDs. Previous studies have evaluated the costs associated with RA in Italy. ${ }^{6,15,16,20-22}$ In a recently published literature review ${ }^{22}$ that looked at the economic burden of RA in Italy, the mean annual social cost (considering overall direct plus indirect costs) of RA was reported to be $€ 13,595$ per adult patient (2012 values). Direct medical costs accounted for $21 \%$ of the total costs, while the remaining 79\% were non-medical costs (direct non-medical costs and indirect costs). ${ }^{22}$

Our study showed that the overall direct annual cost (both RA and non-RA-related costs) amounted to $€ 3,743$ per patient (year value). Of the overall cost, about $44 \%$ was not directly related to RA itself. This proportion is significantly high and may be due to costs incurred in the treatment of comorbidities. A task force of the American College of Rheumatology and the EULAR has recently highlighted the importance of comorbidities in the management of RA and in the treatment decision-making strategies. Evidence suggests that a significant aspect of RA is certainly the presence of comorbidities that may cause a worse health state for the patient, thus influencing the response to therapy and entailing higher expense for their treatment. ${ }^{23-25,27}$ Indeed, data from the latest Social Report issued by the National Association of Rheumatic Patients in Italy $^{27}$ showed that $69.8 \%(n=451)$ of interviewed patients also use drugs to treat other diseases, and this percentage increases with age. These findings suggest that special attention needs to be given to the effective treatment of comorbidities in RA patients, as this may impact overall treatment costs and put a strain on financial resources.

We acknowledge some limitations of our study, principally, due to the descriptive nature of the analysis, based on data collected through administrative claims databases. However, it is real-world observational studies that provide evidence on how specific drugs are used in the market and what impact they have in the long-term on the already limited health resources. This is in contrast to randomized controlled trials where data are limited to the experimental conditions of the trial design, ${ }^{28-31}$ and where results may not translate fully to the real world. ${ }^{28,30}$ Another limitation is represented by the lack of data in the administrative claims databases on clinical outcome measures, such as effectiveness of treatment and disease severity, data on comorbidities and other potential confounders that could have influenced our results. In addition, the reasons for switching and low persistence to treatment, as well as the reasons for drug dose escalation or reduction, are not recorded in the databases. Finally, though our study covered major LHUs in different regions in Italy, there may be a need for further validation using samples of patients from other LHUs. The results and conclusions of this study are limited to the population analyzed.

In conclusion, our study showed that there is still much that needs to be learned about the prescription of csDMARDs 
and biologics to RA patients in Italy and to identify areas for future research. The knowledge of RA management in a real-life clinical setting could offer an opportunity to improve the management of RA in Italy.

\section{Acknowledgments}

This study was funded by Eli Lilly. The views expressed here are those of the authors and not necessarily those of the funders. The abstract of this paper was presented at the International Society for Pharmacoeconomics and Outcome Research (ISPOR) 19th Annual European Congress as a poster presentation. The poster's abstract was published in "Poster Abstracts" Volume 19, Value in Health journal in November 2016.

\section{Disclosure}

WF is an employee of Eli Lilly \& Company, Erl Wood, UK; PLR is an employee of Eli Lilly \& Company, Madrid, Spain; and SL, VR and SA are employees of Eli Lilly Italy S.p.a., Italy. VP, LDE, SD, and SB report no conflicts of interest in this work. Clicon S.r.l. is an independent company. The agreement sign by Clicon S.r.l. and Eli Lilly \& Company does not create any entityship, joint venture or any similar relationship between parties. Neither CliCon nor any of their representatives are employees of Eli Lilly \& Company for any purpose.

\section{References}

1. World Health Organization. Chronic diseases and health promotion. Chronic rheumatic conditions. Available from: http://www.who.int/ chp/topics/rheumatic/en/ Accessed April 12, 2017.

2. Cross M, Smith E, Hoy D, et al. The global burden of rheumatoid arthritis: estimates from the Global Burden of Disease 2010 study. Ann Rheum Dis. 2014;73:1316-1322.

3. Rossini M, Rossi E, Bernardi D, et al. Prevalence and incidence of rheumatoid arthritis in Italy. Rheumatol Int. 2014;34:659-664.

4. Smolen JS, Landewé R, Breedveld FC, et al. EULAR recommendations for the management of rheumatoid arthritis with synthetic and biological disease-modifying antirheumatic drugs: 2013 update. Ann Rheum Dis. 2014:73:492-509.

5. Misra DP, Agarwal V, Sharma A, Wakhlu A, Negi VS. 2016 update of the EULAR recommendations for the management of rheumatoid arthritis: a utopia beyond patients in low/middle income countries? Ann Rheum Dis. Ann Rheum Dis.2017;76:e47.

6. Mennini FS, Marcellusi A, Gitto L, Iannone F. Economic burden of rheumatoid arthritis in Italy: possible consequences on anti-citrullinated protein antibody-positive patients. Clin Drug Investig. 2017;37: 375-386.

7. Agenzia Italiana del Farmaco (AIFA). Guideline for the classification and conduction of the observational studies on medicines. 2010. Available from: https:/www.agenziafarmaco.gov.it/ricclin/sites/default/files/ files_wysiwyg/files/CIRCULARS/Circular\%2031st\%20May\%202010. pdf. Accessed April 12, 2017.

8. Gonnella JS, Lewis DZ, Gozum MV, Callahan CA, Barnes CA. Disease Staging Clinical and Coded Criteria. Version 5.26. Ann Arbor, MI: Thomson Medstat; 2010.
9. Bonafede MM, Gandra SR, Fox KM, Wilson KL. Tumor necrosis factor blocker dose escalation among biologic naive rheumatoid arthritis patients in commercial managed-care plans in the 2 years following therapy initiation. J Med Econ. 2012;15(4):635-643.

10. Darkow T, Chastek B, Rosenblatt L, Digisha T, Hebden T, Henk H, Liu F. dose escalation among rheumatoid arthritis patients treated with infliximab or abatacept: comparison in claims data. Arthritis Rheum. 2011;63:1221; abstract.

11. Neri Serneri GG, di Bartoli V. Sulla eredopatologia delle mes- enchimopatie reattive (cosidette malattie del collageno). II: Ricerche sui fattori ereditari del reumatismo cronico primario [Heredo-pathology of reactive mesenchymal diseases (so-called collagen diseases). II. Researches on hereditary factors of chronic primary rheumatism]. Acta Genet Med Gemellol. 1956;5:402-425. Italian.

12. Marcolongo F, Marcolongo R, Carcassi A, et al. Epidemiologia dell'artrite reumatoide [Epidemiology of reumathoid arthritis]. Siena: U Periccioli Ed; 1967

13. Cimmino MA, Parisi M, Moggiana G, Mela GS, Accardo S. Prevalence of rheumatoid arthritis in Italy: the Chiavari Study. Ann Rheum Dis. 1998;57:315-318.

14. Marotto D, Nieddu ME, Cossu A, et al. Prevalenza dell'artrite reumatoide nel Nord Sardegna: lo studio di Tempio Pausania [Prevalence of rheumatoid arthritis in North Sardinia: the Tempio Pausaniatie reat]. Reumatismo. 2005;57:273-276. Italian.

15. Sangiorgi D, Benucci M, Nappi C, Perrone V, Buda S, Degli Esposti L. Drug usage analysis and health care resources consumption in naïve patients with rheumatoid arthritis. Biol Targets Ther. 2015;9:119-127.

16. Degli Esposti L, Favalli EG, Sangiorgi D, et al. Persistence, switch rates, drug consumption and costs of biological treatment of rheumatoid arthritis: an observational study in Italy. Clin Outcomes Res. 2016;9:9-17.

17. Gaujoux-Viala C, Gossec L, Cantagrel A, et al. Recommendations of the French Society for Rheumatology for managing rheumatoid arthritis. Joint Bone Spine. 2014;81:2874297.

18. Caporali R, Conti F, Alivernini S, et al. Recommendations for the use of biologic therapy in rheumatoid arthritis: update from the Italian Society for Rheumatology I. Efficacy. Clin Exp Rheumatol. 2011;29:S711.

19. Machado MA, Moura CS, Ferré F, Bernatsky S, Rahme E, Acurcio Fde A. Persistência do tratamento em pacientes com artrite reumatoide e espondilite anquilosante [Treatment persistence in patients with rheumatoid arthritis and ankylosing spondylitis]. Rev Saúde Pública. 2016; 50:50. Portugese.

20. Benucci M, Rogai V, Atzeni F, Hammen V, Sarzti-Puttini P, Migliore A. Costs associated with rheumatoid arthritis in Italy: past, present, and future. Clin Outcomes Res. 2016:8:33-41.

21. Leardini G, Salaffi F, Montanelli R, Gerzeli S, Canesi B. A multicenter cost-of-illness study on rheumatoid arthritis in Italy. Clin Exp Rheumatol. 2002;20;505-515.

22. Turchetti G, Bellelli S, Mosca M. The social cost of rheumatoid arthritis in Italy: the results of an estimation exercise. Reumatismo. 2014;65:271.

23. Prevalence of chronic diseases in older Italians: comparing self-reported and clinical diagnoses. The Italian Longitudinal Study on Aging Working Group. Int J Epidemiol. 1997;26:995-1002.

24. Ohinmaa AE, Thanh NX, Barnabe C, et al. Canadian estimates of health care utilization costs for rheumatoid arthritis patients with and without therapy with biologic agents: health care costs for rheumatoid arthritis in Canada. Arthritis Care Res. 2014;66:1319-1327.

25. Dougados M, Soubrier M, Antunez A, et al. Prevalence of comorbidities in rheumatoid arthritis and evaluation of their monitoring: results of an international, cross-sectional study (COMORA). Ann Rheum Dis. 2014:73:62-68.

26. Gullick NJ, Scott DL. Co-morbidities in established rheumatoid arthritis. Best Pract Res Clin Rheumatol. 2011;25:469-483.

27. Censis-ANMAR-SIR Un percorso ad ostacoli. Primo rapporto sociale sull'artrite reumatoide. [An obstacle course. First social report on rheumatoid arthritis]. 2008. Available from: http://www.anmar-italia.it/ cosa-facciamo/appuntamenti/193-qun-percorso-ad-ostacoliq. Accessed April 19, 2017. Italian. 
28. Saturni S, Bellini F, Braiso F, et al. Randomized controlled trials and real life studies. Approaches and methodologies: a clinical point of view. Pulm Pharmacol Ther. 2014:27:129-138.

29. Association of the British Pharmaceutical Industry. Demonstrating value with real world data: a practical guide. 2011. Available from: http:// www.abpi.org.uk/our-work/library/guidelines/Pages/real-world-data. aspx. Accessed April 19, 2017.
30. Garrison LP, Neumann PJ, Erickson P, Marshall D, Mullins CD. Using real-world data for coverage and payment decisions: The ISPOR RealWorld Data Task Force Report. Value Health. 2007;10:326-335.

31. Nallamothu BK, Hayward RA, Bates ER. Beyond the randomized clinical trial: the role of effectiveness studies in evaluating cardiovascular therapies. Circulation. 2008;118:1294-1303.
Open Access Rheumatology: Research and Reviews is an international, peerreviewed, open access journal publishing original research, reports, editorials, reviews and commentaries on all aspects of clinical and experimental rheumatology in the clinic and laboratory including the following topics: Pathology, pathophysiology of rheumatological diseases; Investigation, treatment and management of rheumatological diseases; Clinical trials and novel pharmacologi$\mathrm{cal}$ approaches for the treatment of rheumatological disorders. The manuscript management system is completely online and includes a very quick and fair peer-review system, which is all easy to use. Visit http://www.dovepress.com/ testimonials.php to read real quotes from published authors. 